\title{
Interleukin-28A maintains the intestinal epithelial barrier function through regulation of claudin-1
}

\author{
Liangzi Li, Chao Zhou, Teming Li, Weidong Xiao, Min Yu^, Hua Yang \\ Department of General Surgery, Xinqiao Hospital, Army Medical University, Chongqing, China \\ Contributions: (I) Conception and design: H Yang, M Yu; (II) Administrative support: H Yang, M Yu; (III) Provision of study materials or patients: \\ L Li, T Li, W Xiao; (IV) Collection and assembly of data: L Li, C Zhou, T Li; (V) Data analysis and interpretation: L Li, M Yu; (VI) Manuscript \\ writing: All authors; (VII) Final approval of manuscript: All authors. \\ Correspondence to: Hua Yang; Min Yu. Department of General Surgery, Xinqiao Hospital, Army Medical University, Chongqing 400037, China. \\ Email: huayang@tmmu.edu.cn; yumimianbao@163.com.
}

Background: Interleukin-28A (IL-28A or interferon- $\lambda 2$ ) is reported to maintain intestinal mucosal
homeostasis. However, the effects and mechanisms of IL-28A on intestinal ischemia reperfusion (I/R) have
not yet been studied. Methods: Adult C57BL/6 mice were randomly divided into three groups: sham, I/R, and $\mathrm{I} / \mathrm{R}+\mathrm{IL}-28 \mathrm{~A}$ (n=5 in each group). The I/R+IL-28A group mice were injected with recombinant mouse IL-28A 12 hours before the operation. Mice were sacrificed 6 hours after reperfusion. The mucosal permeability was investigated, and histology analyses were performed. Additionally, a hypoxic Caco-2 cell culture model was established. Fludarabine was used to inhibit phosphorylated signal transducer and activator of transcription 1 (pSTAT1). The expression of IL-28A, tight junctions (TJs), and pSTAT1 was assessed by western blot, immunohistochemical (IHC) staining, or immunofluorescence staining. Epithelial permeability was measured by transepithelial electrical resistance (TER).

Results: The expression of IL-28A was decreased in intestinal lamina propria in the I/R group compared with the control group. Administration of IL-28A significantly alleviated the I/R-induced increase in intestinal permeability and tissue damage. Treatment with IL-28A significantly attenuated intestinal I/ R-induced disruption of TJ proteins, including zonula occludens-1 (ZO-1), occludin, and claudin-1. In vitro, IL-28A treatment reversed the decrease in TER of Caco-2 monolayers exposed to hypoxic environments. IL-28A led to the activation of STAT1 and the upregulation of claudin-1 expression both in vivo and in vitro. Also, inhibiting phosphorylation of STAT1 reversed the effects of IL-28A on the expression and distribution of claudin-1 in Caco-2 cells.

Conclusions: Intestinal epithelial barrier dysfunction caused by intestinal I/R is ameliorated by IL-28A via the regulation of claudin-1.

Keywords: IL-28A; I/R; intestinal epithelial barrier; STAT1; claudin-1

Submitted Jul 24, 2020. Accepted for publication Dec 10, 2020.

doi: 10.21037/atm-20-5494

View this article at: http://dx.doi.org/10.21037/atm-20-5494

\section{Introduction}

Intestinal ischemia/reperfusion (I/R) occurs frequently with numerous pathological processes, such as trauma, hemorrhagic shock, obstruction of the intestine, small bowel transplantation and other major surgeries (1). Studies have shown that the human small intestine is more

^ ORCID: 0000-0002-8845-8977. 
susceptible to I/R injury than the colon (2). Acute small intestinal I/R induces severe epithelial damage and increases intestinal permeability $(3,4)$. Loss of intestinal epithelial barrier function leads to bacterial translocation, resulting in systemic inflammation and multiple organ failure with high mortality $(5,6)$.

The intestinal epithelial barrier is an important defense barrier between the lumen and the intestinal mucosa. The barrier comprises an intact single-cell layer of intestinal epithelial cells (IECs) and tight junctions (TJs) between the IECs. The TJs are a group of transmembrane proteins, including claudins, occludin, and zonula occludens ( $\mathrm{ZO}$ ), are located at the apical membrane, and regulate the passage of ions, nutrients, and water (7). The underlying mechanisms of intestinal I/R injury are very complicated, and accumulating studies show that intestinal barrier dysfunction plays a critical role in intestinal I/R $(4,6)$. Evidence has suggested that the destruction of TJs' expression or distribution is responsible for the increase in intestinal permeability and dysfunction of the intestinal epithelial barrier caused by I/R $(8,9)$. Therapeutic enhancement of the intestinal TJ barrier has been shown to ameliorate intestinal I/ $\mathrm{R}$ injury development (9). Accordingly, new strategies, especially those targeting the intestinal barrier function, are urgently needed to treat intestinal I/R.

Interferon (IFN)- $\lambda$, also known as type III IFN, was first identified in $2003(10,11)$. Type III IFNs include four members in humans, IFN- $\lambda 1 /$ interleukin (IL)-29, IFN- $\lambda 2$ / IL-28A, IFN- $\lambda 3 / \mathrm{IL}-28 \mathrm{~B}$, and IFN- $\lambda 4$, and two members in mice (IFN- $\lambda 2 / \mathrm{IL}-28 \mathrm{~A}, \mathrm{IFN}-\lambda 3 / \mathrm{IL}-28 \mathrm{~B})$ (12). There is a close relationship between IL-28A and IL-28B, and they share approximately $96 \%$ sequence identity (13). The receptor complex of IFN- $\lambda$ comprises the specific subunit IFN- $\lambda$ receptor chain 1 (IFNLR1 or IL-28R1) and IL-10 receptor chain 2 (IL-10R2), which is shared by the receptors of some type II cytokines. IL-28R1 is expressed mainly by epithelial cells of barrier sites, such as the skin, respiratory tract, liver, gastrointestinal tract and blood-brain barrier, which leads to the functional tissue-specificity of IFN- $\lambda$ (14). Previous studies have shown that IFN- $\lambda$ plays a vital role in the clearance of viral infections at barrier sites, including the viruses hepatitis C (15-17), hepatitis B (18), herpes simplex 1 (19), influenza $(20,21)$, rhinovirus (22) and rotavirus (23). Also, IFN- $\lambda$ can provide therapeutic benefits in some autoimmune diseases, including rheumatoid arthritis (24), psoriasis (25), and experimental asthma $(26,27)$.
A recent study showed that IL-28A controls the proliferation of IECs in mice with colitis and accelerates intestinal mucosal healing (28). Additionally, the administration of exogenous IL-28 can tighten the endothelial junctions in the blood-brain barrier (14). However, little is known about the role of IL-28A in intestinal epithelial barrier function and the pathogenesis of intestinal I/R. Using in vivo acute intestinal I/R models, our study demonstrated that IL-28A has a vital role in protecting against intestinal $\mathrm{I} / \mathrm{R}$ injury and maintaining epithelial barrier function. Moreover, we found that IL$28 \mathrm{~A}$ regulated the expression and distribution of claudin-1 in a phosphorylated signal transducer and activator of transcription 1 (pSTAT1)-dependent manner both in vivo and in vitro.

We present the following article in accordance with the ARRIVE reporting checklist (available at http://dx.doi. org/10.21037/atm-20-5494).

\section{Methods}

\section{Animals}

Male C57BL/6 mice (6-8 weeks old), weighing 17-23 g, were purchased from the Laboratory Animal Center of the Army Medical University in Chongqing, China. The mice were fed and housed in temperature-, humidity-, and light-controlled conditions. All animal-related experiments complied with guidelines for the care and use of laboratory animals of the Animal Care and Use Committee at the Army Medical University, and the protocols were approved by the Laboratory Animal Welfare and Ethics Committee of the Third Military Medical University (SYXK 20170002).

\section{Induction of intestinal I/R and IL-28A treatment}

The mice were randomized into a sham group, I/R group, and $\mathrm{I} / \mathrm{R}+\mathrm{IL}-28 \mathrm{~A}$ group ( $\mathrm{n}=5$ in each group). All mice fasted for 12 hours before the operation. The I/R+IL-28A group mice were given intraperitoneal injections of recombinant murine IL-28A (0.5 $\mu \mathrm{g} / \mathrm{g}$ body weight; Peprotech, Rocky Hill, NJ, USA) 12 hours before the operation. All mice were anesthetized with intraperitoneal injections of pentobarbital, and a laparotomy was performed under aseptic conditions. For the I/R and I/R+IL-28A groups, traumatic microvascular clamps were used to occlude the superior mesenteric artery (SMA) of each mouse for 20 minutes. The mice in the sham group underwent the 
same procedures except for SMA occlusion. Then, the clamps were removed, and the incisions were closed. The mice were sacrificed 6 hours after intestinal reperfusion. The small intestinal tissues were quickly collected for histological examination and protein extraction.

\section{Detection of intestinal permeability}

Intestinal permeability was detected by fluorescein isothiocyanate (FITC)-dextran 3,000-5,000 (Sigma, St. Louis, MO, USA). Briefly, the mice were gavaged with FITC-dextran at $0.5 \mathrm{mg} / \mathrm{g}$ body weight after I/R. The blood of the mice was collected and centrifuged after 3 hours. The concentration of FITC-dextran in the serum was quantified by detecting the emission wavelength at $520 \mathrm{~nm}$ using a microplate reader (Varioskan Flash; Thermo Scientific, Waltham, MA, USA).

\section{Histological examination}

After the mice were sacrificed, the small bowels were obtained, and their segments were washed and fixed in $4 \%$ paraformaldehyde. Then, the tissues were dehydrated in ethanol and embedded in paraffin. The sections were stained with hematoxylin \& eosin (H\&E).

\section{Cell isolation}

After the mice were sacrificed, the small bowels were collected, washed with phosphate-buffered saline (PBS), and cut into small fragments. The fragments were then incubated in Hanks' balanced salt solution (HBSS) containing 10\% fetal bovine serum (FBS), $5 \mathrm{mM}$ ethylenediaminetetraacetic acid (EDTA), and $1 \mathrm{mM}$ dithiothreitol (DTT) on a shaker for 30 minutes at $37^{\circ} \mathrm{C}$. The IECs were purified from the supernatant by Percoll (Cell Signaling Technology, Shanghai, China) gradient centrifugation. The remaining fragments were incubated in Roswell Park Memorial Institute (RPMI) 1,640 medium with $0.5 \mathrm{mg} / \mathrm{mL}$ DNaseA (Sigma, St. Louis, MO, USA) and $250 \mathrm{U} / \mathrm{mL}$ collagenase (Sigma) on a shaker for 30 minutes at $37^{\circ} \mathrm{C}$. Intestinal lamina propria cells were collected from the supernatant.

\section{Immunobistochemistry staining}

The segments of mouse jejunum were fixed in $4 \%$ paraformaldehyde overnight, dehydrated, embedded in paraffin, and then cut into $5 \mathrm{~mm}$ sections. The tissues were deparaffinized and treated with $0.3 \%$ hydrogen peroxide for 10 minutes to block endogenous peroxidase activity. The sections were incubated with rabbit anti-ZO-1 antibody (1:50; Proteintech Biotechnology, Rosemont, IL, USA), anti-occludin antibody (1:50; Proteintech Biotechnology, Rosemont, IL, USA) and anti-claudin-1 antibody (1:50; Proteintech) at $4{ }^{\circ} \mathrm{C}$ overnight. Afterward, the sections were consecutively incubated with biotinylated goat antirabbit IgG (Beyotime Biotechnology, Jiangsu, China) for 30 minutes and streptavidin-enzyme conjugate for 10 minutes at room temperature. The peroxidase activities were detected by diaminobenzidine until the desired staining intensity appeared. After counterstaining with hematoxylin, immunohistochemical (IHC) staining was examined by a light microscope.

\section{Immunofluorescence analysis}

The Caco-2 cell monolayers were cultured in laser confocal microscopy dishes and treated as described below. The cells in the dishes were fixed with $4 \%$ paraformaldehyde at room temperature for 10 minutes, washed 3 times in PBS, and then permeabilized by $0.3 \%$ Triton $\mathrm{X}-100$ in PBS for 20 minutes. After blocking in $3 \%$ bovine serum albumin (BSA) at room temperature for 1 hour, the monolayers were incubated with rabbit anti-claudin-1 (1:50; Abcam, Cambridge, UK) overnight at $4{ }^{\circ} \mathrm{C}$. The monolayers were washed 3 times in PBS and incubated with $\mathrm{Cy} 3-$ conjugated goat anti-rabbit secondary antibody (1:500; Beyotime Biotechnology, Jiangsu, China) at $37^{\circ} \mathrm{C}$ for 1 hour. After washing 3 times in PBS, the monolayers were incubated with 4'6-diamidino-2-phenylindole (DAPI) for 10 minutes to stain the nuclei. Control experiments were performed without primary antibodies to avoid nonspecific staining. After extensive washing, the monolayers were coated with antifade gelatin and analyzed by a confocal laser scanning microscope (Leica TCS SP5, Leica, Wetzlar, Germany). The histofluorescence procedure was similar to that described above. The primary antibodies used for the tissues were mouse anti-IL-28 (1:100; Santa Cruz Biotechnology, Dallas, TX, USA) and rabbit antipSTAT1 (1:50; Cell Signaling, Shanghai, China). The secondary antibodies were FITC-labeled anti-mouse (1:500; Beyotime Biotechnology, Jiangsu, China) and Cy3conjugated goat anti-rabbit secondary antibodies (1:500; 
Beyotime Biotechnology, Jiangsu, China).

\section{Cell culture}

The human intestinal epithelial Caco-2 cell line was purchased from American Type Culture Collection (ATCC, Manassas, VA, USA). The cells were cultured in minimum essential media (MEM) basic medium (Gibco; Thermo Fisher Scientific, Waltham, MA, USA) containing 20\% fetal calf serum (Gibco; Thermo Fisher Scientific, Waltham, MA, USA), $1 \%$ nonessential amino acids (HyClone; GE Healthcare Life Sciences, Chicago, IL, USA), $100 \mathrm{U} / \mathrm{mL}$ streptomycin, and $100 \mathrm{U} / \mathrm{mL}$ penicillin. (Beyotime Biotechnology, Jiangsu, China). The cells were cultured in a humidified $37^{\circ} \mathrm{C}, 5 \% \mathrm{CO}_{2}$ incubator. The cells were subcultured by partial digestion with $0.25 \%$ trypsin and $0.53 \mathrm{mM}$ EDTA in PBS. The medium was refreshed every other day.

\section{Cell treatments}

We used an in vitro hypoxic environment to simulate I/R conditions. Caco-2 cells were grown on Transwell chambers (Corning Costar, Corning, NY, USA) at a $5 \times 10^{5}$ cells/chamber density. When the monolayers reached $60 \%$ confluence, the cells were cultured overnight in MEM basic medium without serum, and were then subjected to a hypoxic environment $\left(1 \% \mathrm{O}_{2}\right.$ and $\left.5 \% \mathrm{CO}_{2}\right)$ for 12 hours with or without recombinant human IL-28A (100 nM; Peprotech, Rocky Hill, NJ, USA) stimulation. The controls were incubated under normoxic conditions. To inhibit pSTAT1 signaling, fludarabine (Selleck, Houston, TX, USA) was used at appropriate concentrations with or without recombinant human IL-28A.

\section{Measurement of transepithelial electrical resistance (TER)}

Caco-2 cells were plated on Transwell chambers with a $0.4 \mu \mathrm{m}$ pore size and grown for several days to form cell monolayers. After the cell monolayers were formed, the experiments were performed, and TER was measured by a Millicell ERS-2 voltohmmeter (Millipore, Burlington, MA, USA) as previously described (29). The results were normalized to the initial values and are shown as percentages.

\section{Western immunoblot analysis}

Isolated mouse intestinal cells and Caco-2 cells were lysed with radioimmunoprecipitation assay (RIPA) lysis buffer (Beyotime Biotechnology, Jiangsu, China) supplemented with phenylmethylsulfonyl fluoride (PMSF) and phosphatase inhibitor (Beyotime Biotechnology, Jiangsu, China). The samples were sonicated for 5 minutes and centrifuged at $15,000 \mathrm{rpm}$ for 20 minutes at $4{ }^{\circ} \mathrm{C}$. The proteins were quantified using a bicinchoninic acid (BCA) protein assay kit (Beyotime) and boiled for 10 minutes after mixing with a protein loading buffer. The samples were separated on a $10 \%$ sodium dodecyl sulfate polyacrylamide gel electrophoresis (SDS-PAGE) gel and then transferred to a polyvinylidene fluoride (PVDF) membrane (Millipore, Burlington, MA, USA). After blocking in 5\% BSA at room temperature for 2 hours, the membranes were incubated overnight at $4{ }^{\circ} \mathrm{C}$ with primary antibodies as follows: anti-IL-28 (1:500; Santa Cruz, Dallas, TX, USA), antiZO-1 (1:800; Proteintech, Biotechnology, Rosemont, IL, USA), anti-occludin (1:800; Proteintech Biotechnology), anti-claudin-1 (1:800; Proteintech Biotechnology), antiSTAT1 (1:1,000; Cell Signaling), anti-pSTAT1 (1:1,000; Cell Signaling), and anti-glyceraldehyde 3-phosphate dehydrogenase (GAPDH) (1:1,000; Boster Biotechnology, Pleasanton, CA, USA). After washing 3 times in trisbuffered saline and Tween 20 (TBST), the membranes were incubated with peroxidase-conjugated secondary antibodies (1:5,000; Boster Biotechnology, Pleasanton, CA, USA) for 1 hour at room temperature. The protein blots were visualized with chemiluminescent horseradish peroxidase (HRP) substrate (Millipore, Burlington, MA, USA) on a Station 4000R (Kodak, Rochester, NY, USA).

\section{Statistical analyses}

All data were analyzed using the statistical software SPSS 20.0 (IBM SPSS Statistics, Chicago, IL, USA). The results are expressed as the means with standard deviation (SD). Comparisons were made with Student's t-tests or oneway analysis of variance (ANOVA). $\mathrm{P}<0.05$ was considered statistically significant.

\section{Results}

\section{Intestinal I/R decreases the expression of IL-28}

The intestinal I/R model was developed to explore the expression of IL-28. First, we evaluated the cellular sources of IL-28 in I/R and control mice by immunofluorescence. We found that the cells expressing IL-28 were mainly in the 
A

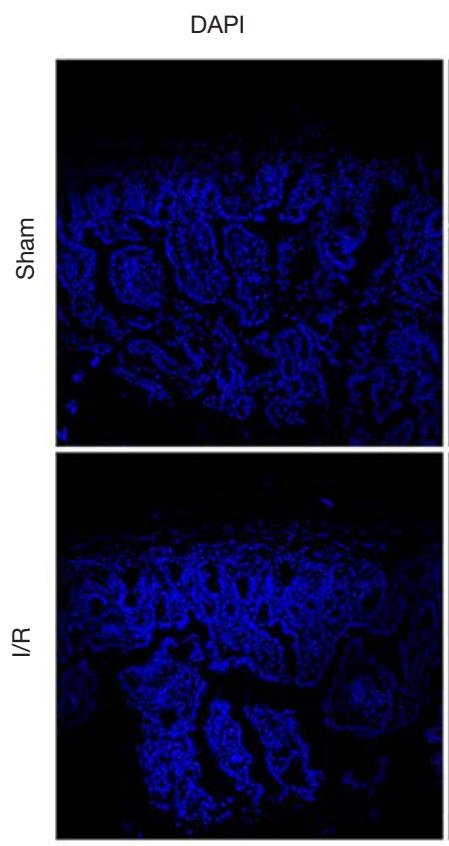

B

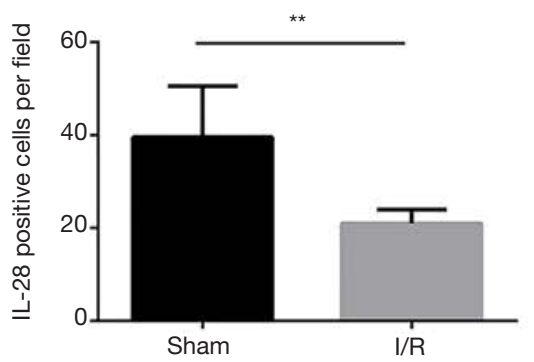

IL-28

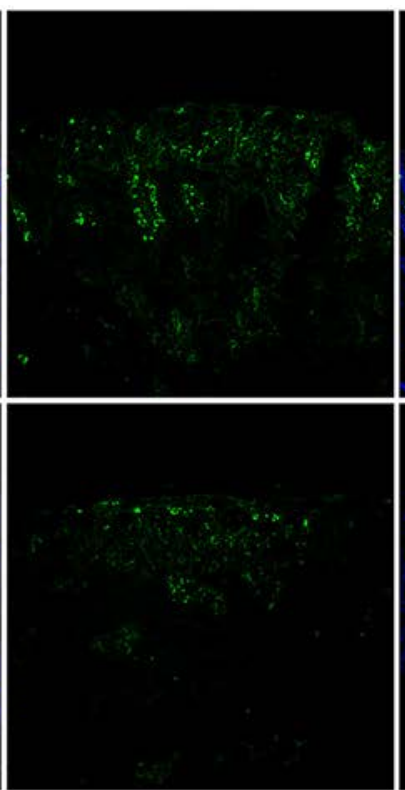

C

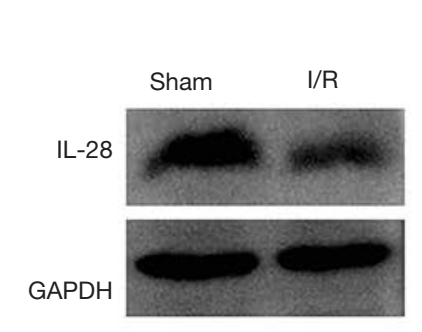

Merge
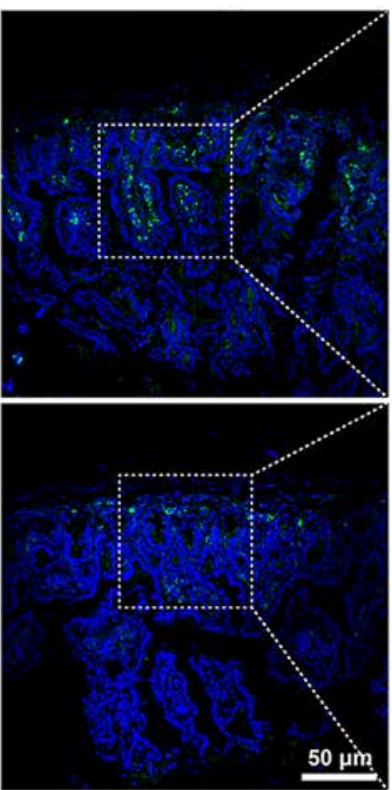

Magnification
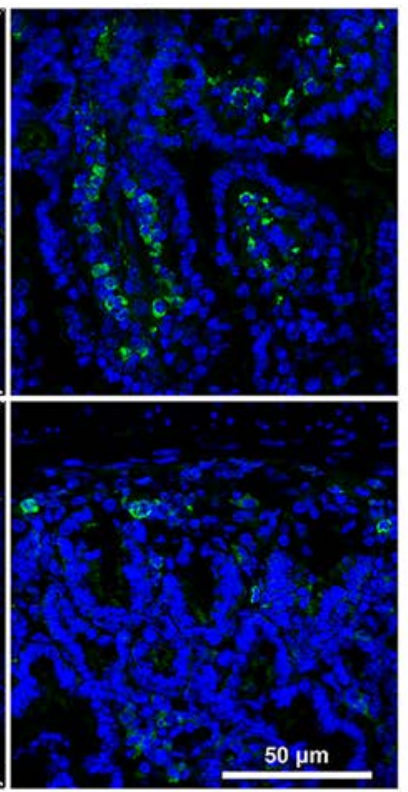

$\mathrm{D}$

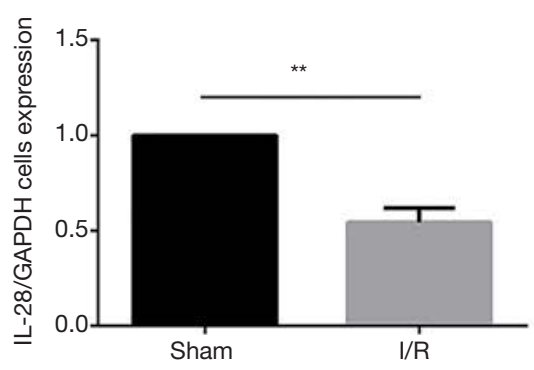

Figure 1 Decreased expression of IL-28 in intestinal I/R. (A) IL-28 (green) expression in the sham and I/R groups was detected by immunofluorescence. Nuclei were counterstained with DAPI. Scale bar $=50 \mu \mathrm{m}$. (B) IL-28-positive cells in high-power fields were counted. The data are shown as the mean with SD $(\mathrm{n}=5)$. ${ }^{* *}, \mathrm{P}<0.01$. (C) Expression of IL-28 was measured by western blot. GAPDH served as a loading control. (D) Relative expression of IL-28 was analyzed by densitometry. The data are shown as the mean with SD $(\mathrm{n}=3) .{ }^{* *}, \mathrm{P}<0.01$. DAPI, 4'6-diamidino-2-phenylindole; GAPDH, glyceraldehyde 3-phosphate dehydrogenase; SD, standard deviation.

lamina propria, and I/R significantly decreased the number of IL-28-positive cells (Figure 1A,B).

Also, western blot analysis showed that the expression of IL-28 protein in the small bowel after intestinal I/ $\mathrm{R}$ was decreased compared with that of the sham group (Figure 1C,D). These observations showed that I/R treatment caused downregulation of IL-28 in the intestines of mice.

\section{IL-28A protects against intestinal I/R injury}

The mice were given injections of recombinant mouse IL-28A for 12 hours before intestinal I/R treatment. We found that IL-28A pretreatment attenuated intestinal I/ $\mathrm{R}$-induced edema and hyperemia of the intestinal tract (Figure 2A). The histological examination showed that I/ $\mathrm{R}$ caused short villi, shedding of the epithelial surface, loss of entire crypts, and immune cell infiltration, while IL-28A pretreatment attenuated these effects (Figure 2B). According to the evaluation criterion developed by Chiu et al. (30), the intestinal damage scores also showed that IL-28A pretreatment relieved the injuries caused by $\mathrm{I} / \mathrm{R}$ (Figure 2C).

To confirm the role of IL-28A in intestinal barrier function, the mice were gavaged with FITC-dextran after the operation. Intestinal I/R led to an increased FITC- 
A

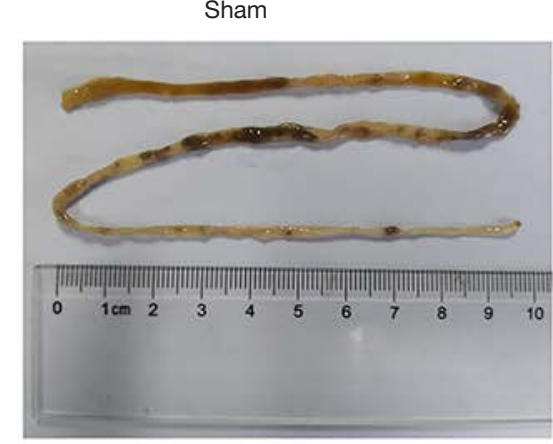

B

C
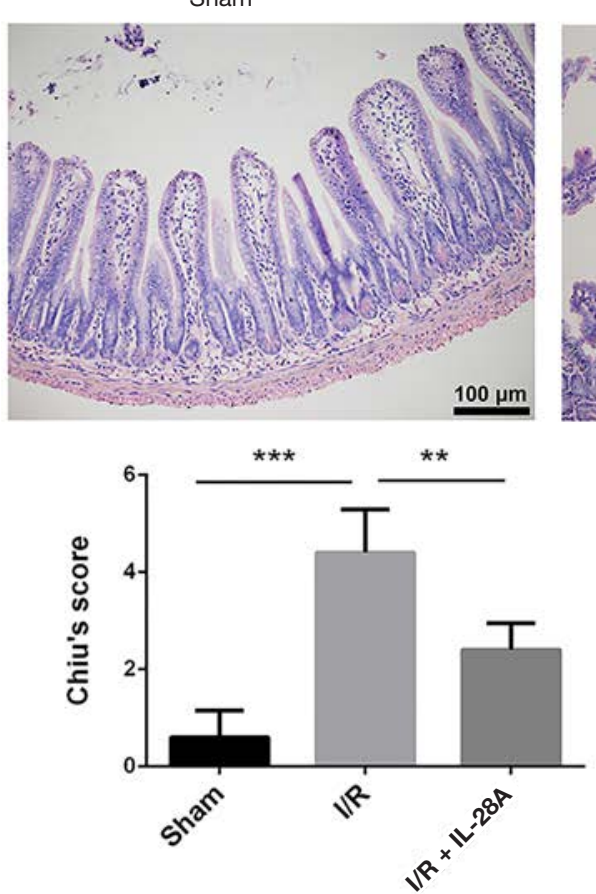

I/R

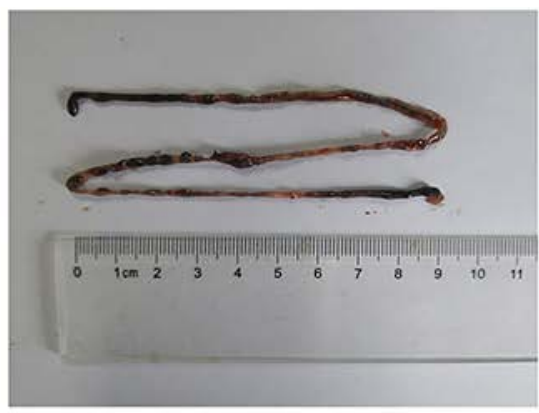

I/R

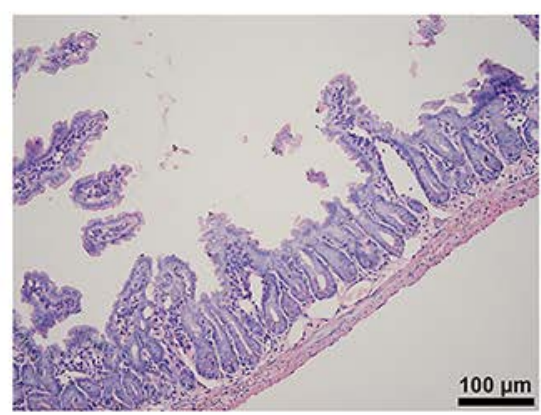

$\mathrm{I} / \mathrm{R}+\mathrm{IL}-28 \mathrm{~A}$

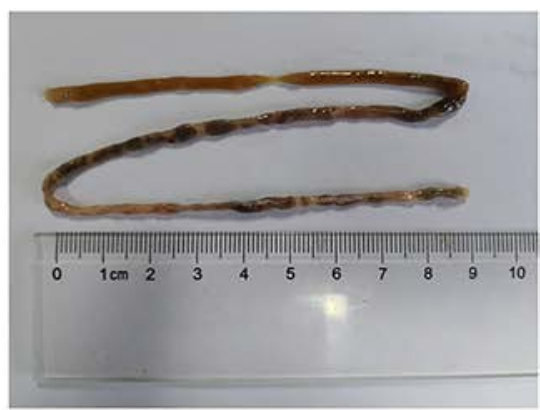

$\mathrm{I} / \mathrm{R}+\mathrm{IL}-28 \mathrm{~A}$

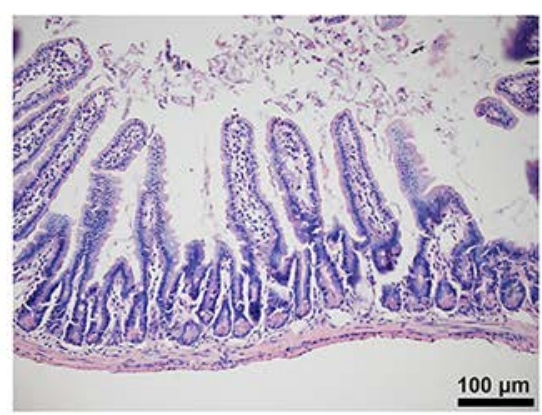

$100 \mu \mathrm{m}$
Figure 2 IL-28A relieves intestinal damage and hyperpermeability caused by I/R. (A) Representative images of small intestines of the 3 groups (sham, I/R, and I/R+IL-28A). (B) HE staining of small intestinal tissues. Scale bar $=100 \mu \mathrm{m}$. (C) Intestinal tissue damage was graded by Chiu's score. The data are presented as the mean with $\mathrm{SD}(\mathrm{n}=5)$. ${ }^{* *}, \mathrm{P}<0.01$, and ${ }^{* * *}, \mathrm{P}<0.001$. (D) Intestinal permeability was detected by FITC-dextran levels in serum. The data are shown as the mean with SD $(n=3)$. ** ${ }^{*}<0.01$. HE, hematoxylin and eosin; FITC, fluorescein isothiocyanate; SD, standard deviation.

dextran level in the serum compared with that of the sham group, while IL-28A pretreatment partially rescued this effect (Figure 2D). These results suggest that IL-28A maintains intestinal barrier function and relieves intestinal damage caused by I/R.

\section{$I L-28$ A regulates the distribution and expression of $T \mathcal{F}$ proteins in intestinal $I / R$}

TJs' disruption is thought to be associated with intestinal epithelial barrier dysfunction caused by enteric diseases (7). Our previous study found that I/R caused an altered distribution and expression of $\mathrm{TJ}$ proteins, leading to the disruption of the intestinal epithelial barrier $(4,9)$. Therefore, we explored the effects of IL-28A on the distribution and expression of TJ proteins, including ZO-1, occludin and claudin-1, by IHC staining and western blotting. The TJ proteins ZO-1, occludin, and claudin-1 were localized at the apexes of intestinal villi in the sham group. The distribution of ZO-1, occludin, and claudin-1 
A
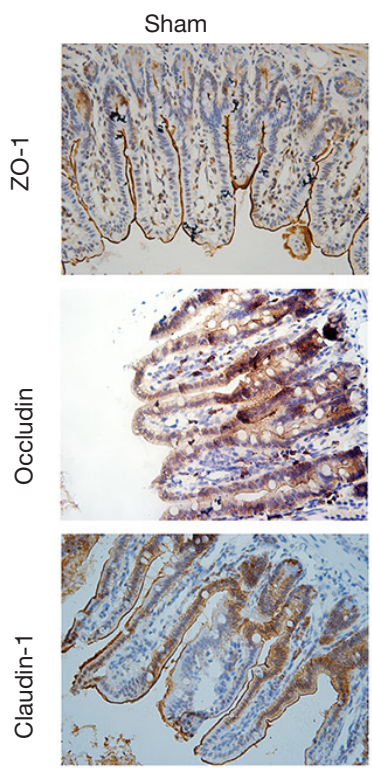

C

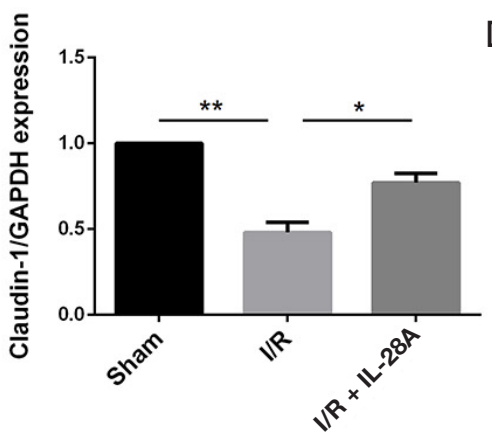

I/R
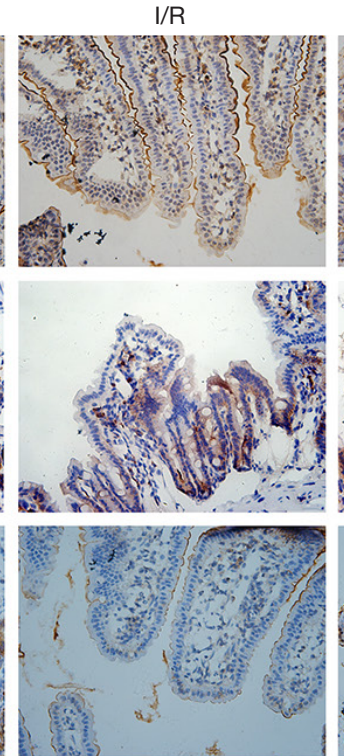

$\mathrm{I} / \mathrm{R}+\mathrm{IL}-28 \mathrm{~A}$
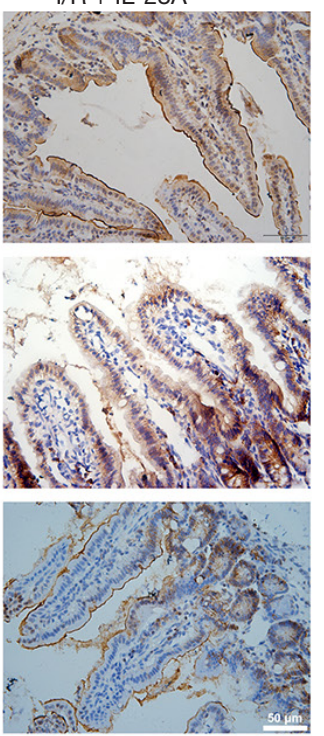

B

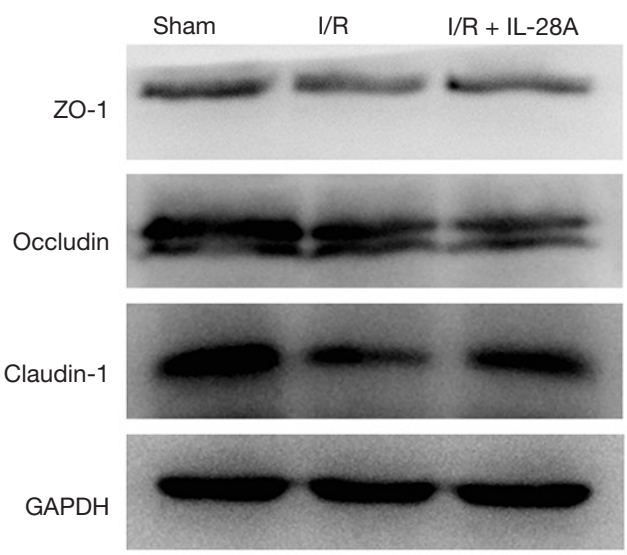

E

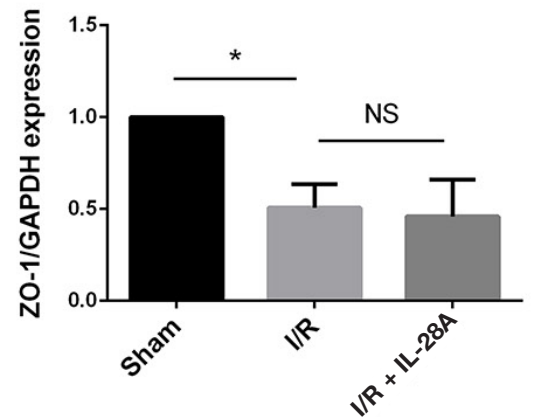

Figure 3 IL-28A maintains the distribution and expression of tight junctions in I/R. (A) The TJ proteins ZO-1, occludin and claudin-1 in the sham, I/R and I/R+IL-28A groups were analyzed by immunohistochemistry staining. Scale bar =50 $\mu \mathrm{m}$. (B) The protein levels of ZO1, occludin, claudin-1, and GAPDH were detected by western blot. GAPDH served as a loading control. (C,D,E) Relative expression of proteins was analyzed by densitometry. The data are shown as the mean with $\mathrm{SD}(\mathrm{n}=3)$. *, $\mathrm{P}<0.05$ and **, $\mathrm{P}<0.01$; NS, not significant. TJ, tight junction; GAPDH, glyceraldehyde 3-phosphate dehydrogenase; SD, standard deviation.

was disrupted in the $\mathrm{I} / \mathrm{R}$ group, while IL-28A pretreatment ameliorated their disruptions (Figure $3 A$ ).

Also, the expression of ZO-1, occludin, and claudin-1 was markedly decreased in I/R group compared with that of the sham group. However, IL-28A prevented the reduction in claudin-1, although it had no effect on the expression of ZO-1 and occludin (Figure 3B,C,D,E). These data suggest that IL-28A ameliorates I/R-induced intestinal epithelial barrier dysfunction by regulating the distribution of TJs and maintaining the expression of claudin-1.

\section{IL-28A protects the epithelial barrier and upregulates the expression of claudin-1 in Caco-2 monolayers}

To further investigate the impact of IL-28A on the intestinal epithelial barrier and the expression of $\mathrm{TJ}$ proteins, human intestinal epithelial Caco-2 cells were cultured and subjected to hypoxic treatment in vitro. Caco-2 cells were incubated with IL-28A in normal or hypoxic conditions for 12 hours. We found that hypoxia decreased the TER of the Caco-2 cell monolayers, whereas IL-28A alleviated this effect. Also, IL-28A administration up-regulated the TER of cells under 
normoxia (Figure 4A).

Next, we examined the role of IL-28A on the expression of TJ proteins in Caco-2 cells. Western blot analysis showed that hypoxia decreased the expression of ZO-1, occludin, and claudin-1. Although it did not affect ZO-1 and occludin expression, IL-28A treatment up-regulated the expression of claudin-1 both under normoxic and hypoxic conditions (Figure $4 B, C, D, E$ ). Subsequently, the localization of claudin-1 in Caco-2 cells was examined by immunofluorescence staining. We found that claudin-1 was mainly expressed on the membrane of Caco-2 monolayers with a continuous belt-like structure under normoxic conditions and that IL-28A treatment increased the staining intensity. After being hypoxic for 12 hours, claudin-1 localization became discontinuous, and the intensity was decreased, while IL-28A treatment ameliorated these changes (Figure 4F). Overall, IL-28A was found to maintain epithelial barrier function by regulating the expression and distribution of claudin-1 in vitro.

\section{IL-28A induces STAT1 phosphorylation both in vivo and in vitro}

STAT1 is an important IL-28A inducible transcription factor and was reported to control intestinal mucosal healing (28). Here, we explored the effect of IL-28A on the activation of STAT1. We found that there was almost no expression of pSTAT1 in mice in the sham or I/R groups, while IL-28A treatment strongly increased pSTAT1 levels in intestinal epithelial cells (Figure $5 A, B, C$ ). Also, IL-28A stimulation induced the phosphorylation of STAT1 in Caco-2 cells, both under normoxic and hypoxic conditions (Figure 5D,E).

\section{Blocking STAT1 phosphorylation decreases the expression of claudin-1 and counteracts the IL-28A-mediated upregulation of claudin-1 expression}

To explore whether IL-28A regulates the expression of claudin-1 and maintains the epithelial barrier function through pSTAT1, we used fludarabine, a pSTAT1 inhibitor, to block the activation of STAT1. We found that although STAT1 expression was largely unaffected, STAT1 phosphorylation was obviously decreased by fludarabine treatment in a concentration-dependent manner in Caco-2 cells (Figure 6A,B). Next, we investigated the effect of fludarabine on the expression of TJ proteins. The results showed that fludarabine successfully inhibited STAT1 phosphorylation with or without IL-28A treatment (Figure 6C,D). Furthermore, the expression of claudin-1 was decreased following pSTAT1 expression, although the expression of ZO-1 and occludin was not altered (Figure 6E,F,G,H). Also, immunofluorescence staining showed the destructive effects of fludarabine on the expression of claudin-1 (Figure 61).

These data indicate that activation of STAT1 increases the expression of claudin-1, and IL-28A protects intestinal epithelial barrier function and upregulates claudin-1 expression through the activation of STAT1.

\section{Discussion}

Intestinal I/R induces intestinal mucosal injury and gut barrier dysfunction, triggering subsequent bacterial translocation, systemic inflammation, multiple organ failure, and eventually death (31). However, the pathophysiological mechanisms of intestinal I/R are not completely understood, and there is no efficient treatment.

This study investigated the potential effects of IL-28A in intestinal $\mathrm{I} / \mathrm{R}$ injury and hypoxia-challenged Caco-2 cells. We confirmed that IL-28A plays important roles in relieving intestinal epithelial injuries and maintaining gut barrier function during murine intestinal I/R. Furthermore, IL-28A led to the activation of STAT1 and up-regulated claudin-1 expression, both in vivo and in vitro. Inhibiting pSTAT1 signaling by fludarabine reversed the effects of IL-28A on the expression and distribution of claudin- 1 in Caco-2 cells. These results suggest that IL-28A regulates TJs and protects intestinal barrier function in a pSTAT1 signaling-dependent pathway.

An abundance of IL-28 is reported to be mainly at barrier surfaces such as the respiratory tract, liver, gastrointestinal tract, and blood-brain barrier. It is robustly expressed by epithelia and epithelial-origin cells such as hepatocytes and certain immune cells (23,32-34). Our study found that intestinal IL-28A was mainly expressed in the lamina propria and was obviously decreased in I/R. Previous studies have indicated that IL-28 conferred antiviral responses at epithelial barrier surfaces $(35,36)$. Also, studies had discovered regulatory roles of IL-28 during chronic inflammation or autoimmune diseases when viral infections were absent, such as collagen-mediated arthritis (24), experimental allergic asthma (27), and T cell-mediated hepatitis (37). Additionally, a recent study indicated that IL-28A regulates gut homeostasis and promotes pSTAT1dependent mucosal wound healing in inflammatory bowel 
A

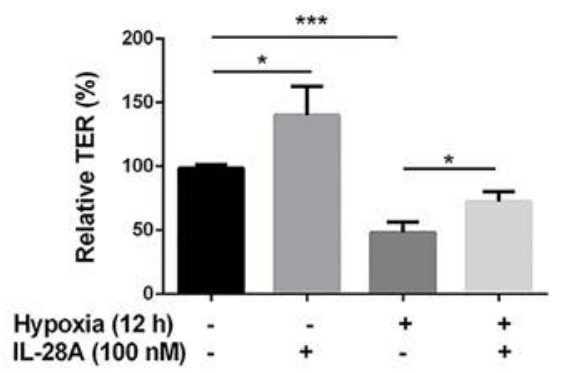

C

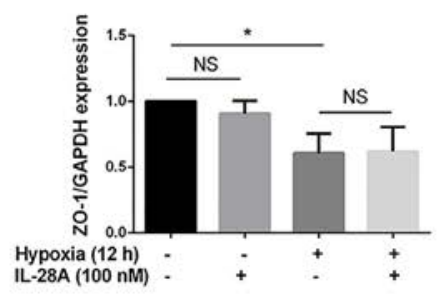

B

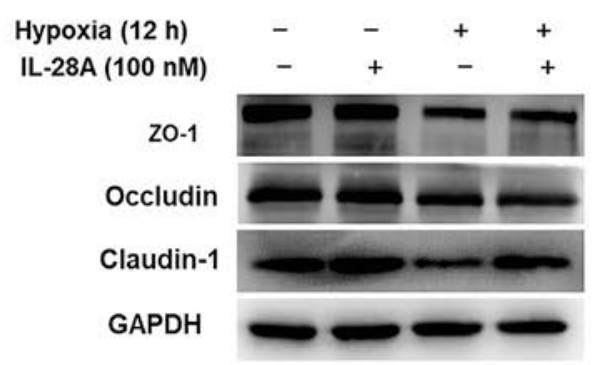

E

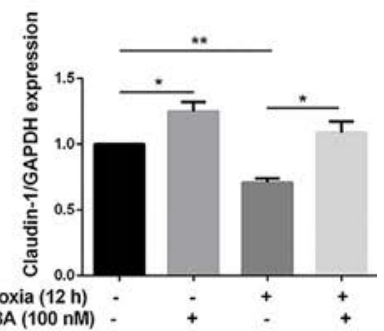

$\mathrm{F}$

Control

D

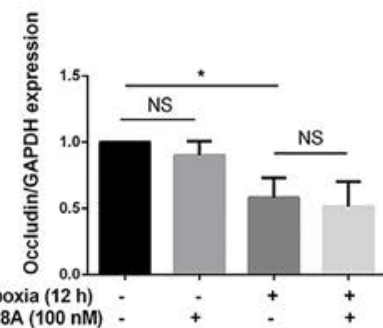

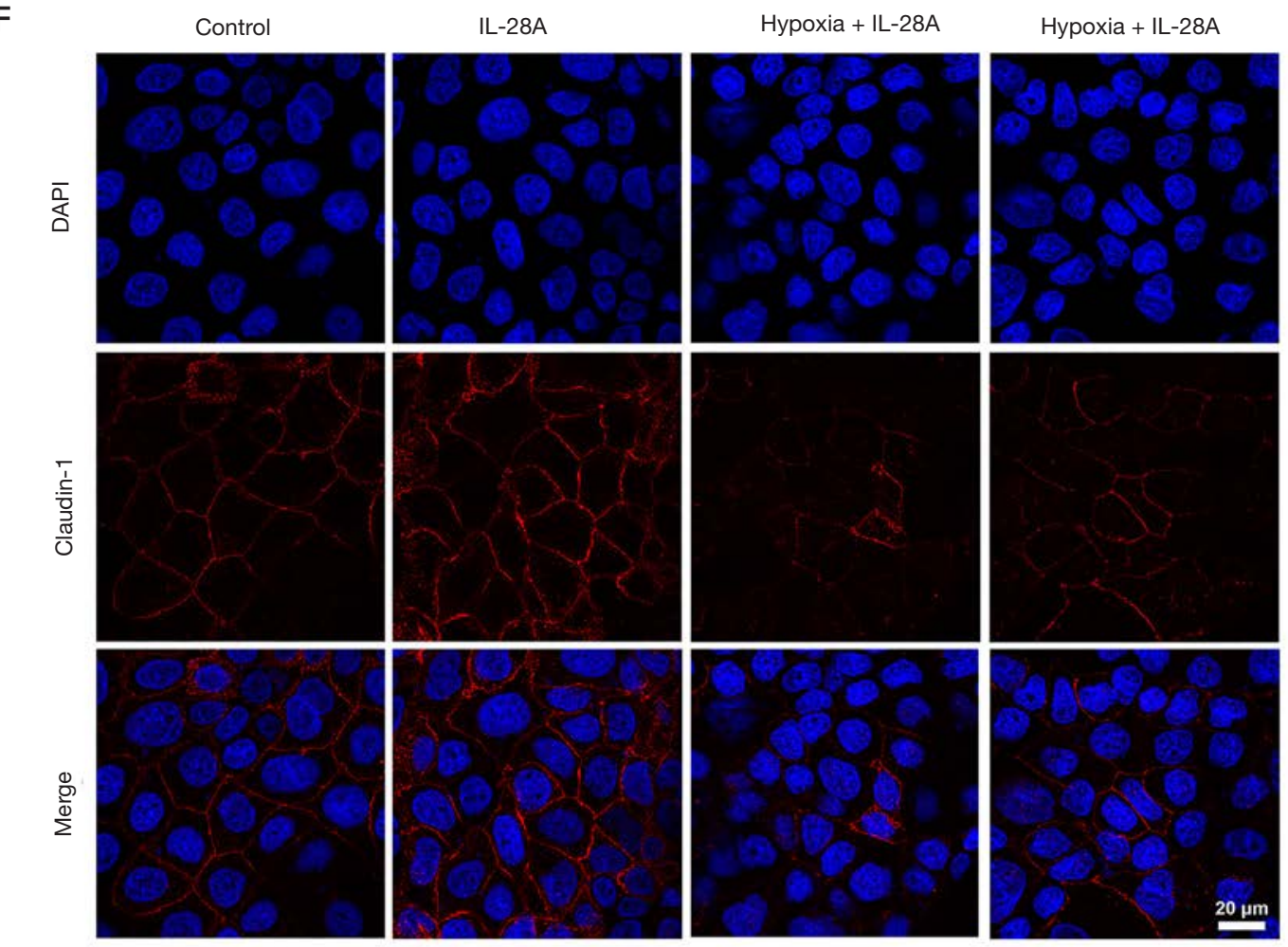

Figure 4 The effects of IL-28A on the expression and localization of TJ proteins in vitro. Caco-2 cells were treated with IL-28A (100 nM) in normal or hypoxic conditions for 12 hours. (A) TER was detected. The data are shown as the means with $\mathrm{SD}(\mathrm{n}=3)$. * $\mathrm{P}<0.05$, and ${ }^{* * *}$, $\mathrm{P}<0.001$. (B) The protein levels of ZO-1, occludin, claudin-1, and GAPDH were detected by western blot. GAPDH served as a loading control. (C-E) Relative expression of proteins was analyzed by densitometry. The data are shown as the means with SD ( $\mathrm{n}=3)$. *, $\mathrm{P}<0.05$ and ${ }^{* *}, \mathrm{P}<0.01$; NS, not significant. (F) Claudin-1 (red) in Caco-2 cells treated as described was detected by immunofluorescence. Nuclei were counterstained with DAPI. Scale bar $=20 \mu \mathrm{m}$. TJ, tight junction; TER, transepithelial electrical resistance; GAPDH, glyceraldehyde 3-phosphate dehydrogenase; SD, standard deviation; DAPI, 4'6-diamidino-2-phenylindole. 
A
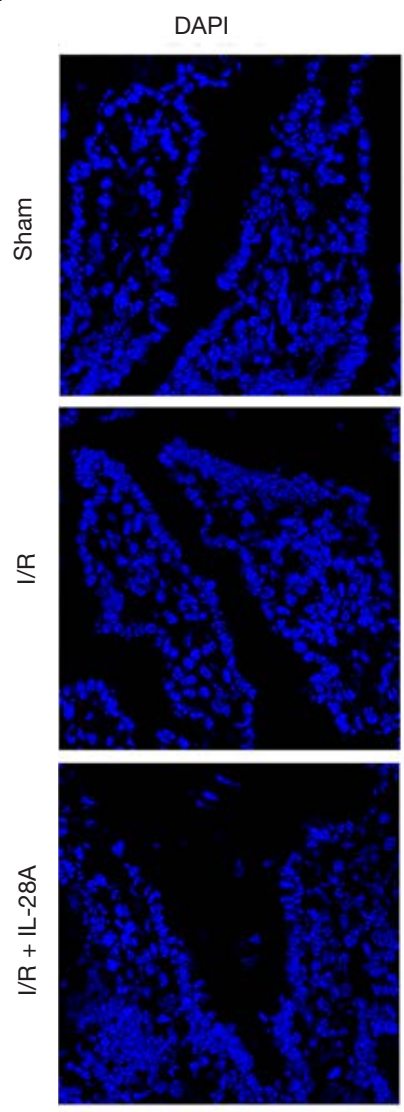

PSTAT1
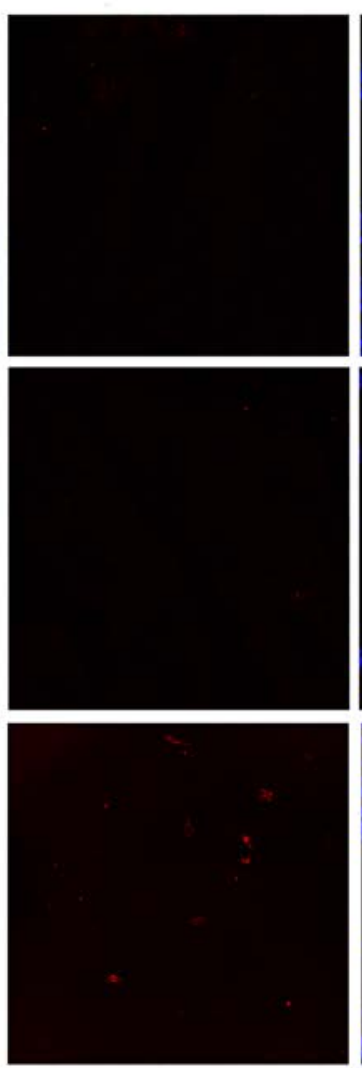
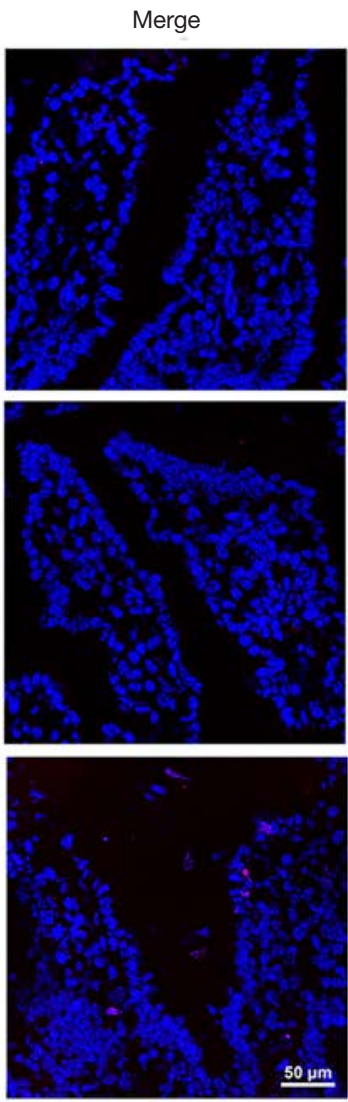

B

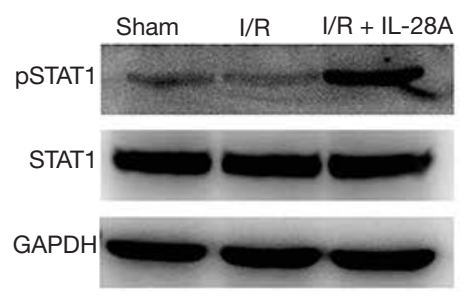

C

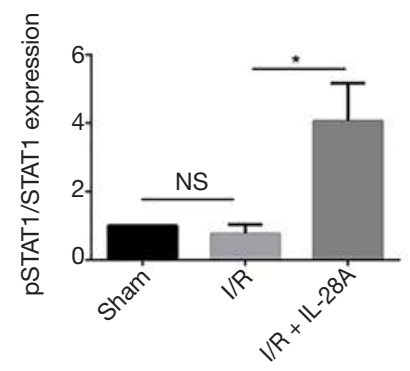

Hypoxia (12 h) $\quad-\quad+\quad-\quad+\quad+\quad+$

IL-28A (100 nM)

pSTAT1

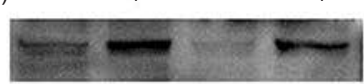

STAT1

GAPDH

E

Figure 5 IL-28A induces STAT1 phosphorylation. (A) pSTAT1 (red) in mice was detected by immunofluorescence. Nuclei were counterstained with DAPI. Scale bar $=50 \mu \mathrm{m}$. (B) The protein levels of pSTAT1, STAT1, and GAPDH in mice were detected by western blot. GAPDH served as a loading control. (C) Relative expression of pSTAT1 was analyzed by densitometry. The data are shown as the means with SD ( $\mathrm{n}=3)$. *, $\mathrm{P}<0.05$; NS, not significant. (D) The protein levels of pSTAT1, STAT1, and GAPDH in Caco-2 cells treated as described were detected by western blot. (E) Relative expression of pSTAT1 was analyzed by densitometry. The data are shown as the means with SD ( $\mathrm{n}=3) .{ }^{* *}, \mathrm{P}<0.01$; NS, not significant. DAPI, 4'6-diamidino-2-phenylindole; GAPDH, glyceraldehyde 3-phosphate dehydrogenase.

disease (IBD) (28). Our study found that exogenous IL$28 \mathrm{~A}$ pretreatment relieved the changes caused by I/R. To our knowledge, this is the first study to demonstrate the protective role of IL-28A in intestinal mucosal morphology during acute intestinal $\mathrm{I} / \mathrm{R}$.

The intestinal epithelial barrier is critical for the maintenance of intestinal homeostasis (7). Studies indicate that disruption of intestinal barrier function has pathological effects on certain gastrointestinal diseases including IBD $(38,39)$, coeliac disease $(40,41)$, and intestinal I/R $(42,43)$. Recent studies have shown that IL-28 exerts protective effects on the airway epithelial and blood-brain 
A

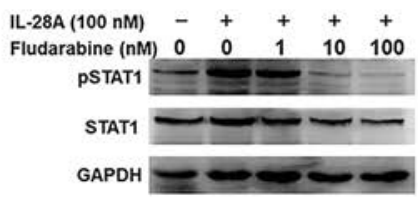

E

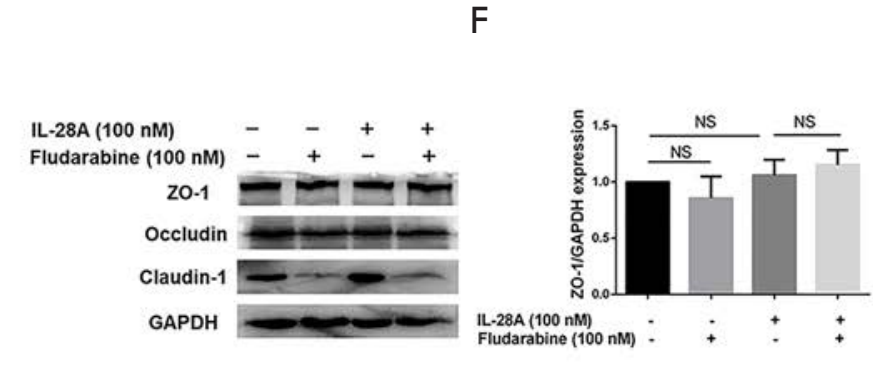

I
B

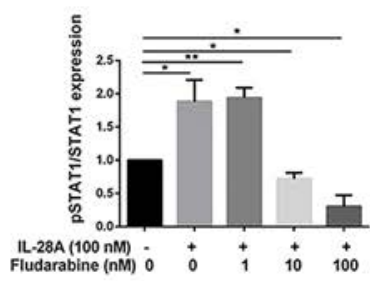

G

C
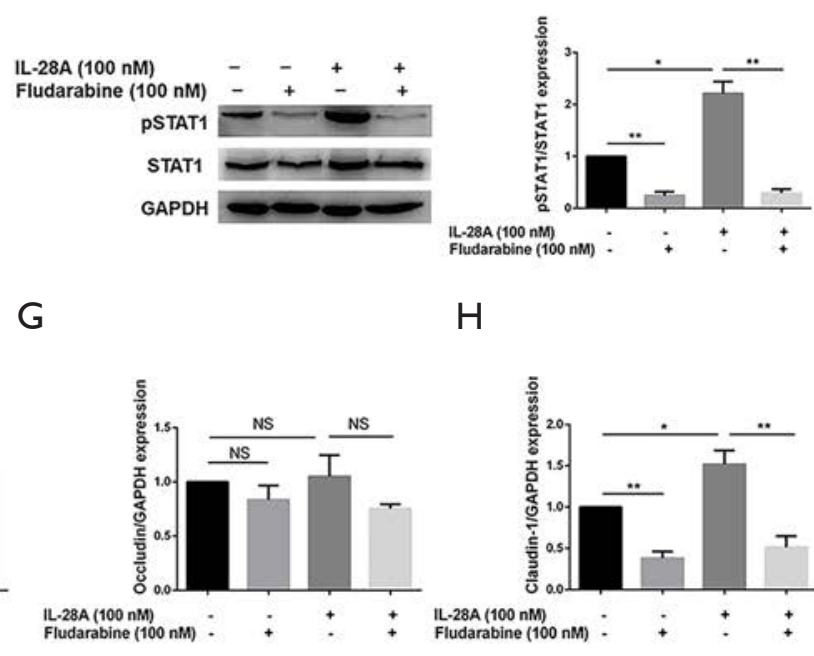

$\mathrm{H}$

D

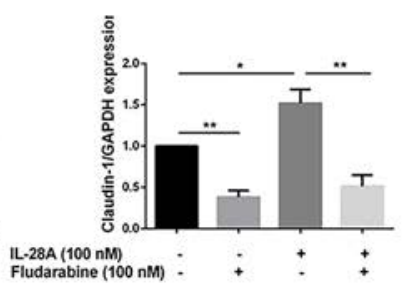

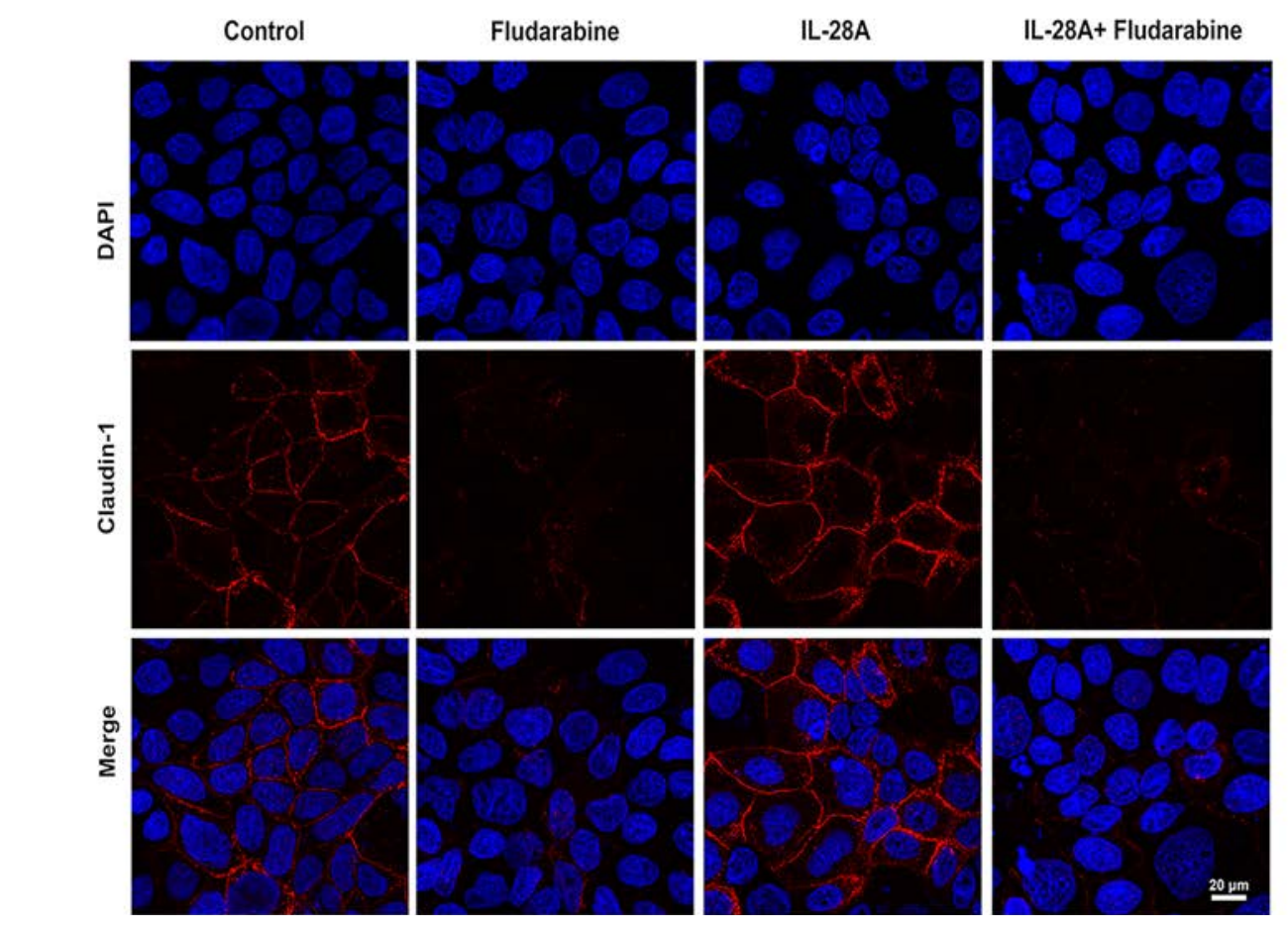

Figure 6 IL-28A up-regulates claudin-1 expression via the pSTAT1 pathway. Caco-2 cells were treated with different amounts of fludarabine $(0,1,10$, and $100 \mathrm{nM})$ with or without IL-28A (100 nM) for 12 hours. (A) The protein levels of pSTAT1, STAT1 and GAPDH were detected by western blot. GAPDH served as a loading control. (B) Relative expression of pSTAT1 was analyzed by densitometry. The data are shown as the means with SD $(\mathrm{n}=3)$. *, $\mathrm{P}<0.05$ and ${ }^{* *}, \mathrm{P}<0.01$. Caco-2 cells were treated with IL-28A (100 nM) with or without fludarabine $(100 \mathrm{nM})$ for 12 hours. (C,E) The protein levels of pSTAT1, STAT1, ZO-1, occludin, claudin-1, and GAPDH were detected by western blot. GAPDH served as a loading control. (D,F-H) Relative expression of pSTAT1, ZO-1, occludin, and claudin-1 was analyzed by densitometry. The data are shown as the means with $\mathrm{SD}(\mathrm{n}=3) .{ }^{*}, \mathrm{P}<0.05$ and ${ }^{* *}, \mathrm{P}<0.01$; NS, not significant. (I) Claudin-1 (red) in Caco-2 cells treated as described was detected by immunofluorescence. Nuclei were counterstained with DAPI. Scale bar $=20 \mu \mathrm{m}$. DAPI, 4'6-diamidino-2-phenylindole; GAPDH, glyceraldehyde 3-phosphate dehydrogenase. 
barriers $(44,45)$. Also, IL-28 has been reported to protect the epithelial barrier function from salmonella infection in T84 cells (46). In the present study, we found that IL$28 \mathrm{~A}$ also had a critical role in maintaining intestinal barrier function during acute intestinal I/R. Also, IL-28A enhanced epithelial barrier function both under normal and hypoxic conditions in Caco-2 cells.

TJ proteins play important roles in maintaining intestinal epithelial barrier function. Intestinal epithelial barrier dysfunction is usually associated with the destruction of $\mathrm{TJ}$ proteins in various intestinal diseases, including intestinal I/R (7,9). A previous study showed that IL-28A could enhance the colocalization of ZO-1 and claudin-5 in endothelial cells (45). In this study, we found that intestinal I/R destroyed ZO-1 and occludin distribution, which was consistent with our previous research. Pretreatment with IL-28A ameliorated the disrupted distribution of ZO-1 and occludin in vivo. However, IL-28A pretreatment did not affect ZO-1 and occludin expression in vivo and in vitro. Interestingly, IL-28A pretreatment up-regulated the expression of claudin-1, both in vivo and in vitro. The results showed that IL-28A protected the intestinal epithelial barrier through the regulation of claudin-1.

Stimulation of IL-28A leads to the activation of Janus kinases (JAK) and induces phosphorylation of the transcription factors STAT1 and STAT2, which is called the canonical JAK-STAT pathway (47). A recent study showed that the expression of STAT2 was low in primary IECs and that IL-28A mediated biological effects mainly by the pSTAT1 signaling pathway in IECs (28). Activation of STAT1 was previously reported to have a wide range of roles, such as suppressing tumors, resisting viral infection, and inducing inflammation (48). In the present study, we found that IL-28A strongly induced phosphorylation of STAT1, both in vivo and in vitro, while inhibiting STAT1 signaling by fludarabine treatment counteracted the effects of IL-28A on the expression of claudin-1 in Caco-2 cells. Furthermore, the inhibition of STAT1 phosphorylation almost completely inhibited the expression of claudin-1, although it did not affect the expression of ZO-1 and occludin. These results suggest that IL-28A upregulates the expression of claudin-1 and maintains intestinal epithelial barrier function through the pSTAT1 pathway.

However, there were still some limitations in the present study. Our study mainly focused on animal and cell culture models and lacked research on clinical samples. The potential of applying IL-28A in intervention for intestinal I/ $\mathrm{R}$ injury needs further exploration.
In conclusion, our study confirmed that IL-28A regulates intestinal epithelial TJs and maintains epithelial barrier function both in an intestinal I/ $\mathrm{R}$ injury model and a hypoxia-challenged Caco-2 cell model. The protective roles of IL-28A in the intestinal barrier are through, or at least partly through, the pSTAT1 signaling pathway. These findings may provide new insight into the management of intestinal I/R.

\section{Acknowledgments}

Funding: This research was supported by grants from the National Natural Science Foundation of China (NSFC 81970468 to MY and NSFC 81873551 to HY), and the Department of the Ministry of Education of China (IRT_17R16 to HY).

\section{Footnote}

Reporting Checklist: The authors have completed the ARRIVE reporting checklist. Available at http://dx.doi. org/10.21037/atm-20-5494

Data Sharing Statement: Available at http://dx.doi. org/10.21037/atm-20-5494

Conflicts of Interest: All authors have completed the ICMJE uniform disclosure form (available at http://dx.doi. org/10.21037/atm-20-5494). The authors have no conflicts of interest to declare.

Ethical Statement: The authors are accountable for all aspects of the work in ensuring that questions related to the accuracy or integrity of any part of the work are appropriately investigated and resolved. All animalrelated experiments complied with guidelines for the care and use of laboratory animals of the Animal Care and Use Committee at the Army Medical University, and the protocols were approved by the Laboratory Animal Welfare and Ethics Committee of the Third Military Medical University (SYXK 20170002).

Open Access Statement: This is an Open Access article distributed in accordance with the Creative Commons Attribution-NonCommercial-NoDerivs 4.0 International License (CC BY-NC-ND 4.0), which permits the noncommercial replication and distribution of the article with the strict proviso that no changes or edits are made and the 
original work is properly cited (including links to both the formal publication through the relevant DOI and the license). See: https://creativecommons.org/licenses/by-nc-nd/4.0/.

\section{References}

1. Mallick IH, Yang W, Winslet MC, et al. Ischemiareperfusion injury of the intestine and protective strategies against injury. Dig Dis Sci 2004;49:1359-77.

2. Hundscheid IH, Grootjans J, Lenaerts K, et al. The Human Colon Is More Resistant to Ischemia-reperfusioninduced Tissue Damage Than the Small Intestine: An Observational Study. Ann Surg 2015;262:304-11.

3. Gonzalez LM, Moeser AJ, Blikslager AT. Animal models of ischemia-reperfusion-induced intestinal injury: progress and promise for translational research. Am J Physiol Gastrointest Liver Physiol 2015;308:G63-75.

4. Yang Y, Qiu Y, Wang W, et al. Adenosine A2B receptor modulates intestinal barrier function under hypoxic and ischemia/reperfusion conditions. Int J Clin Exp Pathol 2014;7:2006-18.

5. Feinman R, Deitch EA, Watkins AC, et al. HIF-1 mediates pathogenic inflammatory responses to intestinal ischemiareperfusion injury. Am J Physiol Gastrointest Liver Physiol 2010;299:G833-43.

6. Carden DL, Granger DN. Pathophysiology of ischaemiareperfusion injury. J Pathol 2000;190:255-66.

7. Odenwald MA, Turner JR. The intestinal epithelial barrier: a therapeutic target? Nat Rev Gastroenterol Hepatol 2017;14:9-21.

8. Li Q, Zhang Q, Wang C, et al. Altered distribution of tight junction proteins after intestinal ischaemia/reperfusion injury in rats. J Cell Mol Med 2009;13:4061-76.

9. Liu Z, Li L, Chen W, et al. Aryl hydrocarbon receptor activation maintained the intestinal epithelial barrier function through Notch1 dependent signaling pathway. Int J Mol Med 2018;41:1560-72.

10. Kotenko SV, Gallagher G, Baurin VV, et al. IFN-lambdas mediate antiviral protection through a distinct class II cytokine receptor complex. Nat Immunol 2003;4:69-77.

11. Sheppard P, Kindsvogel W, Xu W, et al. IL-28, IL-29 and their class II cytokine receptor IL-28R. Nat Immunol 2003;4:63-8.

12. Zanoni I, Granucci F, Broggi A. Interferon (IFN)-lambda Takes the Helm: Immunomodulatory Roles of Type III IFNs. Front Immunol 2017;8:1661.

13. Fox BA, Sheppard PO, O'Hara PJ. The role of genomic data in the discovery, annotation and evolutionary interpretation of the interferon-lambda family. PLoS One 2009;4:e4933.

14. Lazear HM, Nice TJ, Diamond MS. Interferon-lambda: Immune Functions at Barrier Surfaces and Beyond. Immunity 2015;43:15-28.

15. Thomas DL, Thio CL, Martin MP, et al. Genetic variation in IL28B and spontaneous clearance of hepatitis C virus. Nature 2009;461:798-801.

16. Tanaka Y, Nishida N, Sugiyama M, et al. Genomewide association of IL28B with response to pegylated interferon-alpha and ribavirin therapy for chronic hepatitis C. Nat Genet 2009;41:1105-9.

17. Bibert S, Roger T, Calandra T, et al. IL28B expression depends on a novel TT/-G polymorphism which improves HCV clearance prediction. J Exp Med 2013;210:1109-16.

18. Galmozzi E, Vigano M, Lampertico P. Systematic review with meta-analysis: do interferon lambda 3 polymorphisms predict the outcome of interferon-therapy in hepatitis B infection? Aliment Pharmacol Ther 2014;39:569-78.

19. Griffiths SJ, Koegl M, Boutell C, et al. A systematic analysis of host factors reveals a Med23-interferonlambda regulatory axis against herpes simplex virus type 1 replication. PLoS Pathog 2013;9:e1003514.

20. Egli A, Santer DM, O'Shea D, et al. IL-28B is a key regulator of $\mathrm{B}$ - and $\mathrm{T}$-cell vaccine responses against influenza. PLoS Pathog 2014;10:e1004556.

21. Kim HJ, Kim CH, Ryu JH, et al. Reactive oxygen species induce antiviral innate immune response through IFNlambda regulation in human nasal epithelial cells. Am J Respir Cell Mol Biol 2013;49:855-65.

22. Contoli M, Message SD, Laza-Stanca V, et al. Role of deficient type III interferon-lambda production in asthma exacerbations. Nat Med 2006;12:1023-6.

23. Mahlakoiv T, Hernandez P, Gronke K, et al. Leukocytederived IFN-alpha/beta and epithelial IFN-lambda constitute a compartmentalized mucosal defense system that restricts enteric virus infections. PLoS Pathog 2015;11:e1004782.

24. Blazek K, Eames HL, Weiss M, et al. IFN-lambda resolves inflammation via suppression of neutrophil infiltration and IL-1beta production. J Exp Med 2015;212:845-53.

25. Li Y, Cheng H, Zuo XB, et al. Association analyses identifying two common susceptibility loci shared by psoriasis and systemic lupus erythematosus in the Chinese Han population. J Med Genet 2013;50:812-8.

26. Koch S, Finotto S. Role of Interferon-lambda in Allergic Asthma. J Innate Immun 2015;7:224-30.

27. Koltsida O, Hausding M, Stavropoulos A, et al. IL-28A 
(IFN-lambda2) modulates lung DC function to promote Th1 immune skewing and suppress allergic airway disease. EMBO Mol Med 2011;3:348-61.

28. Chiriac MT, Buchen B, Wandersee A, et al. Activation of Epithelial Signal Transducer and Activator of Transcription 1 by Interleukin 28 Controls Mucosal Healing in Mice With Colitis and Is Increased in Mucosa of Patients With Inflammatory Bowel Disease. Gastroenterology 2017;153:123-38.e8.

29. Yu M, Wang Q, Ma Y, et al. Aryl Hydrocarbon Receptor Activation Modulates Intestinal Epithelial Barrier Function by Maintaining Tight Junction Integrity. Int J Biol Sci 2018;14:69-77.

30. Chiu CJ, McArdle AH, Brown R, et al. Intestinal mucosal lesion in low-flow states. I. A morphological, hemodynamic, and metabolic reappraisal. Arch Surg 1970;101:478-83.

31. Vollmar B, Menger MD. Intestinal ischemia/reperfusion: microcirculatory pathology and functional consequences. Langenbecks Arch Surg 2011;396:13-29.

32. Andreakos E, Zanoni I, Galani IE. Lambda interferons come to light: dual function cytokines mediating antiviral immunity and damage control. Curr Opin Immunol 2019;56:67-75.

33. Coccia EM, Severa M, Giacomini E, et al. Viral infection and Toll-like receptor agonists induce a differential expression of type I and lambda interferons in human plasmacytoid and monocyte-derived dendritic cells. Eur J Immunol 2004;34:796-805.

34. Apostolou E, Kapsogeorgou EK, Konsta OD, et al. Expression of type III interferons (IFNlambdas) and their receptor in Sjogren's syndrome. Clin Exp Immunol 2016;186:304-12.

35. Wack A, Terczynska-Dyla E, Hartmann R. Guarding the frontiers: the biology of type III interferons. Nat Immunol 2015;16:802-9.

36. Kotenko SV, Durbin JE. Contribution of type III interferons to antiviral immunity: location, location, location. J Biol Chem 2017;292:7295-303.

37. Siebler J, Wirtz S, Weigmann B, et al. IL-28A is a key regulator of T-cell-mediated liver injury via the T-box transcription factor T-bet. Gastroenterology 2007;132:358-71.

38. Heller F, Florian P, Bojarski C, et al. Interleukin-13 is the key effector Th2 cytokine in ulcerative colitis that affects epithelial tight junctions, apoptosis, and cell restitution.
Gastroenterology 2005;129:550-64.

39. Su L, Nalle SC, Shen L, et al. TNFR2 activates MLCKdependent tight junction dysregulation to cause apoptosis-mediated barrier loss and experimental colitis. Gastroenterology 2013;145:407-15.

40. Sander GR, Cummins AG, Henshall T, et al. Rapid disruption of intestinal barrier function by gliadin involves altered expression of apical junctional proteins. FEBS Lett 2005;579:4851-5.

41. Schumann M, Gunzel D, Buergel N, et al. Cell polaritydetermining proteins Par-3 and PP-1 are involved in epithelial tight junction defects in coeliac disease. Gut 2012;61:220-8.

42. Sun Z, Wang X, Deng X, et al. The influence of intestinal ischemia and reperfusion on bidirectional intestinal barrier permeability, cellular membrane integrity, proteinase inhibitors, and cell death in rats. Shock 1998;10:203-12.

43. McCallion K, Wattanasirichaigoon S, Gardiner KR, et al. Ischemic preconditioning ameliorates ischemia- and reperfusion-induced intestinal epithelial hyperpermeability in rats. Shock 2000;14:429-34.

44. Ferguson SH, Foster DM, Sherry B, et al. Interferonlambda3 Promotes Epithelial Defense and Barrier Function Against Cryptosporidium parvum Infection. Cell Mol Gastroenterol Hepatol 2019;8:1-20.

45. Lazear HM, Daniels BP, Pinto AK, et al. Interferonlambda restricts West Nile virus neuroinvasion by tightening the blood-brain barrier. Sci Transl Med 2015;7:284ra59.

46. Odendall C, Voak AA, Kagan JC. Type III IFNs Are Commonly Induced by Bacteria-Sensing TLRs and Reinforce Epithelial Barriers during Infection. J Immunol 2017;199:3270-9.

47. Odendall C, Kagan JC. The unique regulation and functions of type III interferons in antiviral immunity. Curr Opin Virol 2015;12:47-52.

48. Loh CY, Arya A, Naema AF, et al. Signal Transducer and Activator of Transcription (STATs) Proteins in Cancer and Inflammation: Functions and Therapeutic Implication. Front Oncol 2019;9:48.

Cite this article as: $\mathrm{Li} \mathrm{L}$, Zhou $\mathrm{C}, \mathrm{Li} \mathrm{T}, \mathrm{Xiao} \mathrm{W}, \mathrm{Yu}$ M, Yang H. Interleukin-28A maintains the intestinal epithelial barrier function through regulation of claudin-1. Ann Transl Med 2021;9(5):365. doi: 10.21037/atm-20-5494 